\title{
EPIDEMIOLOGIA DA MORTALIDADE NEONATAL NO CEARÁ NO PERÍODO DE 2005-2015
}

\section{Epidemiology of neonatal mortality in Ceará in the period from 2005-2015 \\ Epidemiología de la mortalidad neonatal en Ceará en el período 2005-2015}

\author{
Ana Nery Melo Cavalcante \\ Universidade de Fortaleza - UNIFOR - Fortaleza (CE) - Brasil \\ Maria Alix Leite Araújo \\ Universidade de Fortaleza - UNIFOR - Fortaleza (CE) - Brasil
}

Shirley Virino Silveira Lopes

Universidade de Fortaleza - UNIFOR - Fortaleza (CE) - Brasil

Tássia Ívila Freitas de Almeida

Hospital Geral de Fortaleza - HGF- Fortaleza (CE) - Brasil

\section{Rosa Lívia Freitas de Almeida}

Universidade de Fortaleza - UNIFOR - Fortaleza (CE) - Brasil

\section{RESUMO}

Objetivo: Analisar os diferenciais da mortalidade infantil, sua distribuição espacial no estado do Ceará e traçar o perfil epidemiológico dos óbitos neonatais. Métodos: Estudo ecológico, tendo os municípios como unidade de análise, que utilizou técnicas de análise espacial e regressão linear para identificar aglomerados espaciais e tendências da mortalidade na série de 2005 a 2015 . Os dados foram coletados no DATASUS, no Sistema de Informação de Mortalidade e no Sistema de Nascidos Vivos, utilizando-se a base cartográfica do estado do Ceará, por município, do Instituto Brasileiro de Geografia e Estatística (IBGE). Resultados: A taxa de mortalidade infantil no período analisado foi de 14,7 por mil nascidos vivos, com redução significativa da linha de tendência com ajuste linear de $63 \%\left(\mathrm{R}^{2}=88 ; p<0,001\right)$, predomínio do componente neonatal precoce $(53,7 \%)$ e distribuição heterogênea da mortalidade infantil entre os municípios. A maioria das crianças era do sexo masculino $(56,3 \%)$, prematuras $(60,7 \%)$, apresentaram baixo peso ao nascer $(64,1 \%)$, nasceram de parto vaginal $(57,6 \%)$, tiveram óbito após o parto $(84,3 \%)$ e como causa do óbito, afecções originadas no período neonatal e malformações congênitas $(96,2 \%)$. Segundo as genitoras, destacou-se a faixa etária de 20 a 34 anos (51,6\%), com escolaridade inferior a 12 anos de estudo (62,8\%). Conclusão: Aglomerados de autocorrelação espacial foram identificados para a mortalidade geral e a neonatal precoce. Apesar da redução significativa da mortalidade no período, alguns municípios apresentaram taxas acima da média do estado.

Descritores: Mortalidade Infantil; Perfil de Saúde; Perinatologia; Atenção Primária à Saúde.

\section{ABSTRACT}

Objective: To analyze the differences in infant mortality and its spatial distribution in the state of Ceará and to identify the epidemiological profile of neonatal deaths. Methods: An ecological study was carried out in the municipalities using spatial analysis and linear regression techniques to identify spatial clusters and mortality trends in the period from 2005 to 2015 . Data were collected from DATASUS, the Mortality Information System, and the Live Births System using a cartographic base of the state of Ceará by municipality developed by the Brazilian Institute of Geography and Statistics (Instituto Brasileiro de Geografia e Estatística - IBGE). Results: The infant mortality rate in the period analyzed was 14.7 per thousand live births and there was a significant reduction in the trend line with linear adjustment of $63 \%\left(R^{2}=88 ; p<0.001\right)$, predominance of the early neonatal component $(53.7 \%)$ and heterogeneous distribution of infant mortality among municipalities. Most of the children were male (56.3\%), premature (60.7\%), had low birth weight (64.1\%), were born vaginally (57.6\%), died after delivery (84.3\%) and the main causes of death were conditions originating in the neonatal period and congenital malformations (96.2\%). As for the mothers, the majority were 20 to 34 years old (51.6\%) and had less than 12 years of study (62.8\%). Conclusion: Clusters of spatial autocorrelation were

Este artigo foi selecionado, corrigido e aprovado para publicação pelo VI Seminário Internacional em Promoção da Saúde, seguindo suas normas e formatação. 
identified for general and early neonatal mortality. Despite the significant reduction in mortality in the period, some municipalities presented rates above the state's average.

Descriptors: Infant Mortality; Health Profile; Perinatology; Primary Health Care.

\section{RESUMEN}

Objetivo: Analizar los diferenciales de la mortalidad infantil, su distribución espacial en el estado de Ceará y trazar el perfil epidemiológico de los óbitos neonatales. Métodos: Estudio ecológico con unidad de análisis en los municipios utilizando técnicas de análisis espacial y regresión linear para la identificación de aglomerados espaciales y tendencias de mortalidad entre 2005 y 2015. Se recogieron los datos en el DATASUS, en el Sistema de Información de Mortalidad y en el Sistema de Nacidos Vivos utilizándose la base cartográfica del estado de Ceará, por municipio, del Instituto Brasileño de Geografía y Estadística (IBGE). Resultados: La tasa de mortalidad infantil en el período analizado fue de 14,7 para cada mil nacidos vivos con reducción significativa de línea de tendencia con ajuste linear del $63 \%\left(R^{2}=88 ; p<0,001\right)$, predominio del componente neonatal precoz (53,7\%) y distribución heterogénea de la mortalidad infantil entre los municipios. La mayoría de los niños era del sexo masculino (56,3\%), prematuros (60,7\%), con bajo peso al nacer (64,1\%), de parto vaginal (57,6\%), con óbito después del parto (84,3\%) y las afecciones originadas del periodo neonatal y las malformaciones congénitas como causa de óbito (96,2\%). Se destacó la franja de edad entre 20 y 34 años (51,6\%) entre las genitoras con escolaridad inferior a 12 años de estudio (62,8\%). Conclusión: Se han identificado aglomerados de auto correlación espacial para la mortalidad general y la neonatal precoz. Pese una reducción significativa de la mortalidad en el período, algunos municipios presentaron tasas por encima de la media del estado.

Descriptores: Mortalidad Infantil; Perfil de Salud; Perinatología; Atención Primaria de Salud.

\section{INTRODUÇÃO}

A taxa de mortalidade infantil (TMI) representa um importante indicador, pois reflete as condições de vida e saúde de uma população. Nas últimas décadas, esse coeficiente apresentou redução em todo o mundo, persistindo taxas elevadas nos países em desenvolvimento( ${ }^{(1)}$. No Brasil, o declínio na TMI ocorreu devido a melhorias das condições socioeconômicas e sanitárias e ao acesso aos serviços de saúde ${ }^{(2)}$.

A TMI é definida pelas mortes de crianças em seu primeiro ano de vida em relação ao número de nascidos vivos para o mesmo período. A Organização Mundial da Saúde (OMS) considera aceitável dez mortes para cada mil nascimentos ${ }^{(3)}$. Essa taxa pode ser analisada segundo dois componentes: mortalidade neonatal, que representa os óbitos de crianças no período de 0 a 27 dias de vida, e a mortalidade pós-neonatal, que são os óbitos de 28 dias até um ano de vida. A mortalidade neonatal, por sua vez, é subdividida em neonatal precoce (óbitos de 0 a 6 dias completos de vida) e neonatal tardia (óbitos de 7 a 27 dias de vida) ${ }^{(4)}$.

Até o final dos anos 80 , ocorreu uma predominância do componente de óbitos pós-neonatais no Brasil e, após esse período, passou a prevalecer o componente neonatal, representando cerca $70 \%$ da mortalidade infantil na atualidade. A redução da mortalidade infantil ocorreu por diversos fatores: queda da fecundidade, maior acesso da população ao saneamento e a serviços de saúde, aumento da prevalência de aleitamento materno, maior abrangência das ações de imunização, e uso de antibióticos e da terapia de reidratação oral(5,6). Contudo, para avançar mais, especialmente para a redução do componente neonatal, o Ministério da Saúde, em parceria com os estados e municípios, vem implantando algumas estratégias, como a Rede Cegonha, reforçando a importância da melhoria da qualidade da assistência ao pré-natal, ao parto e ao recém-nascido ${ }^{(7)}$.

No ano 2000, a ONU determinou metas sociais para os países e fixou prazo até 2015 para que os objetivos de desenvolvimento do milênio fossem atingidos. O ano de 1990 foi a base para comparação. No Brasil, a meta era que as 58 mortes registradas, em 1990, para cada mil crianças nascidas vivas fossem reduzidas para 19 por mil nascidos vivos em 2015. No final de 2011, a taxa já havia sido atingida(8,9).

Segundo a pesquisa Estatística do Registro Civil de 2015, do IBGE, as mortes de crianças com até 1 ano de idade passaram de $4 \%$ do total de óbitos registrados em 2005 para $2,5 \%$ em $2015^{(10)}$.

O Brasil, quando comparado com outros países, apresenta coeficientes altos, ocupando a $107^{a}$ posição na tabela de mortes de crianças, em especial de crianças menores de 5 anos, apesar da redução dos coeficientes de mortalidade infantil e da infância e da tendência de homogeneidade de alguns determinantes sociais. Além disso, os óbitos infantis permaneceram desproporcionalmente concentrados nas regiões mais pobres, representando desigualdade regional. Entre 2000 e 2012, 47,6\% do total de óbitos no país ocorreu no Norte e Nordeste do Brasi(l(11).

No estado do Ceará, a TMI apresentou redução progressiva, semelhante à situação epidemiológica nacional. Foi observada uma redução de $57 \%$ no período de 1997 a 2013, com expressiva queda do componente pós-neonatal 
e estabilização do componente neonatal. Em 2016, a TMI foi de 12,9 por mil nascidos vivos, destacando-se por ser a mais baixa dos últimos anos, entretanto não ocorre uma homogeneidade dentro dos municípios. Dentre os 184 municípios do estado, 24 atingiram TMI superior a 22,8 para cada mil nascidos vivos ${ }^{(12)}$.

Nesse contexto, este estudo teve por objetivo analisar os diferenciais da mortalidade infantil, sua distribuição espacial no estado do Ceará e traçar o perfil epidemiológico dos óbitos neonatais.

\section{MÉTODOS}

Estudo ecológico e descritivo, tendo como unidade de análise os 184 municípios do estado do Ceará, nos anos de 2005 a 2015. Analisou-se a taxa de mortalidade infantil e seu componente neonatal precoce e tardio utilizando técnicas de análise espacial. O trabalho foi desenvolvido com dados do Sistema de Informação de Mortalidade (SIM) e Sistema de Informações de Nascidos Vivos (SINASC), do DATASUS, através do site http://datasus.saude.gov.br/, e a base cartográfica do estado do Ceará, por município, disponibilizada pela Instituto Brasileiro de Geografia e Estatística (IBGE) através do site https://www.ibge.gov.br/.

Inicialmente, realizou-se a análise dos dados para conhecer o perfil epidemiológico do componente neonatal do estado. Foram descritas as variáveis da mãe (escolaridade e faixa etária) e das crianças (sexo, peso e idade gestacional ao nascer, tipo de parto, época do óbito em relação ao parto e causa básica do óbito segundo capítulo do CID-10). Em seguida, para cada município e em cada ano, construíram-se as taxas de mortalidade infantil, neonatal precoce e tardia, e pós-neonatal por mil nascidos vivos.

Foi realizada análise exploratória das TMI geral, neonatal precoce e tardia utilizando o teste de Moran global, apresentando-os em mapas. O índice de Moran global é uma medida de correlação espacial usada para avaliar até que ponto o nível de uma variável para uma determinada área é similar às áreas vizinhas. Varia no intervalo de -1 $a+1$, sendo os valores próximos a zero de ausência de correlação espacial e, quando positivos, indicam existência de áreas com valores similares ${ }^{(13)}$. Realizou-se ainda regressão linear da TMI geral e dos componentes neonatal precoce e tardio para verificar a tendência. Nessa análise, foi utilizado o teste $\mathrm{R}$ de Pearson e todas as funções de distribuição de dados foram testadas. Para todos os testes, adotou-se o alfa abaixo de 0,05 como necessário para rejeição das hipóteses nulas.

Para o perfil epidemiológico, utilizou-se o Programa Microsoft Excel 2013®, pertencente ao pacote Microsoft Office $2013 \AA$, para Windows $\AA$, e os resultados estão dispostos em tabelas com valores relativos e absolutos. $O$ cálculo do índice de Moran global foi executado com o software TerraView, versão 4.2.2® (Instituto Nacional de Pesquisas Espaciais; http: //www.dpi.inp.br/terraview) e utilizou-se o software Qgis-2.18.21® (http: //www.qgisbrasil. org) para os mapas.

\section{RESULTADOS}

No estado do Ceará, no período de 2005 a 2015, foram registrados, no SIM, 21.259 óbitos em menores de um ano, segundo local de residência. Destes, $68,6 \%(n=14.593)$ ocorreram no período neonatal; $78,0 \%(n=11.389)$ ocorreram de 0 a 6 dias de nascido; e 21,9\% ( $n=3.204$ ) ocorreram de 7 a 27 dias. O número de nascidos vivos (NV), considerando o Ceará como o local de residência da mãe, contabilizou 1.444 .568 para o período. A TMI geral foi de 14,7 por mil NV, sendo 7,9 por mil NV para a taxa de mortalidade neonatal precoce e 2,2 por mil NV para a taxa de mortalidade neonatal tardia. O componente neonatal precoce e tardio correspondeu a, respectivamente, $53,7 \%$ e $15 \%$ da TMI no período em estudo.

Observou-se, através de linha de tendência com ajuste linear, uma redução significativa da TMI geral de $63 \%$ $\left(\mathrm{R}^{2}=88 ; p<0,001\right)$. A redução do componente neonatal tardio foi de $7,2 \%\left(\mathrm{R}^{2}=0,70 ; p=0,001\right)$ e do neonatal precoce foi de $26 \%\left(R^{2}=82 ; p<0,001\right)$ (Figura 1$)$.

A Tabela I apresenta o perfil das crianças que evoluíram para óbito no período neonatal.

Quanto ao sexo, $56,3 \%(n=8.225)$ eram do sexo masculino e $42,7 \%(n=6.224)$ eram do sexo feminino. Segundo a idade gestacional (IG), 60,7\% ( $n=8.848$ ) nasceram com menos de 37 semanas e $23,7 \%(n=3.460)$ nasceram a termo (maiores ou igual a 37 semanas). Segundo o peso, $64,1 \%(n=9.336)$ apresentaram peso menor que $2.500 \mathrm{~g}$ (baixo peso ao nascer) e destes, 30,2\% ( $n=4.404)$ evoluíram para óbito com peso inferior a 1000 gramas (extremo baixo peso) (Tabela I).

Sobre o nascimento, $57,6 \%(n=8.410)$ nasceram de parto vaginal e o óbito aconteceu após o parto em $84,3 \%$ $(n=12.299)$ dos casos (Tabela I). Quanto à causa do óbito, segundo capítulos do CID-10, a maioria, 96,2\% ( $n=14.041)$, foi devido a algumas afecções originadas no período neonatal e a malformações congênitas. 
A Tabela II apresenta o perfil das genitoras cujos filhos evoluíram para óbito com menos de um ano. Foi observado que a maioria estava na faixa etária de 20 a 34 anos, 51,6\% $(n=7.5335)$. Em relação à escolaridade, $62,8 \%(n=9.161)$ apresentaram menos de 12 anos de estudo.

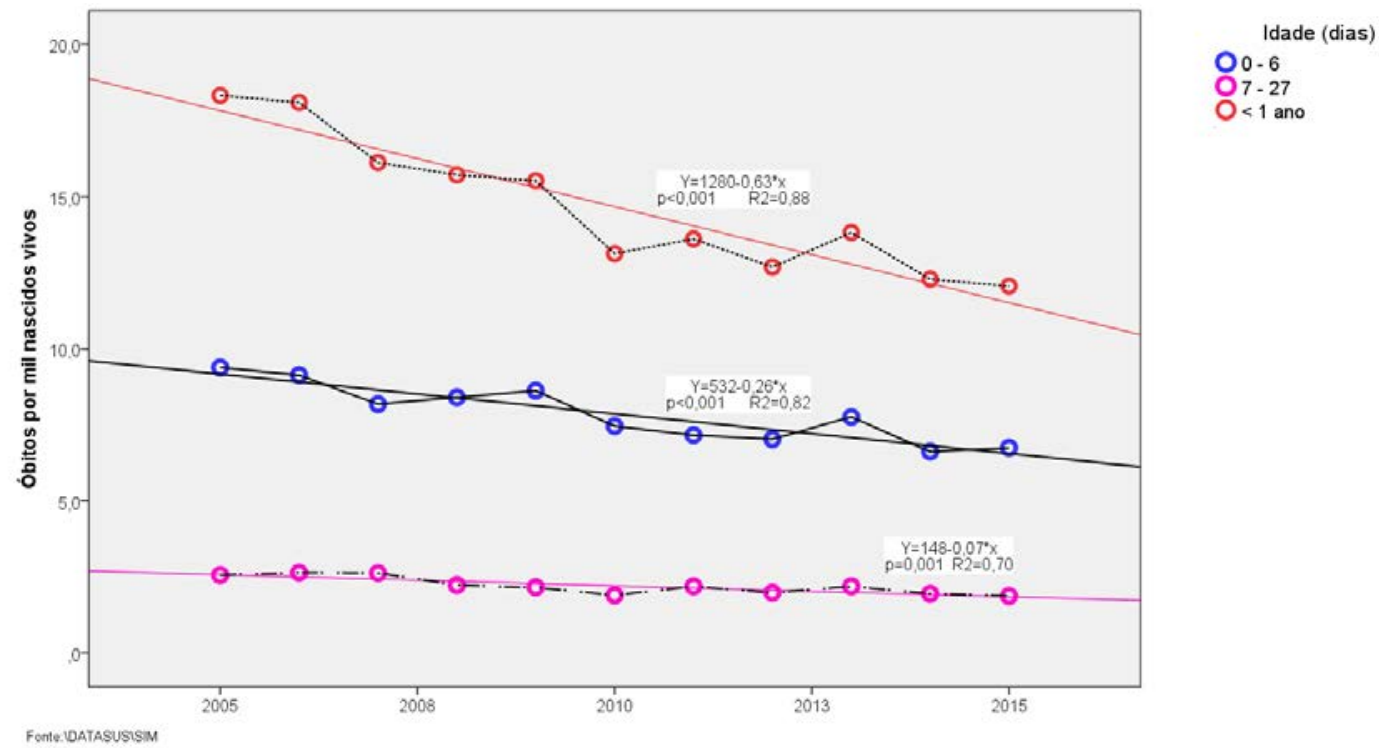

Figura 1 - Representação gráfica da regressão linear da taxa de mortalidade infantil e de seus componentes neonatal precoce e tardio. Ceará, 2005-2015.

Tabela I - Faixa etária, sexo, peso ao nascer, duração da gestação e causa básica do óbito em crianças no período neonatal. Ceará, 2005-2015.

\begin{tabular}{|c|c|c|}
\hline Variáveis neonatais & $n=14593$ & $\%$ \\
\hline \multicolumn{3}{|l|}{ Sexo } \\
\hline Masculino & 8225 & 56,3 \\
\hline Feminino & 6224 & 42,7 \\
\hline Ignorado & 144 & 1,0 \\
\hline \multicolumn{3}{|l|}{ Peso ao nascer } \\
\hline$<500$ & 872 & 6,0 \\
\hline 500 a 999 & 3532 & 24,2 \\
\hline 1000 a 1499 & 1874 & 12,9 \\
\hline 1500 a 2499 & 3058 & 21,0 \\
\hline 2500 a 2999 & 1365 & 9,3 \\
\hline 3000 a 3999 & 1929 & 13,2 \\
\hline 4000 ou mais & 238 & 1,6 \\
\hline Ignorado & 1725 & 11,8 \\
\hline \multicolumn{3}{|c|}{ Duração da gestação (semanas) } \\
\hline$<22$ & 835 & 5,8 \\
\hline 22 a 27 & 2901 & 19,9 \\
\hline 28 a 31 & 2159 & 14,8 \\
\hline 32 a 36 & 2953 & 20,2 \\
\hline 37 a 41 & 3341 & 22,9 \\
\hline$>42$ & 119 & 0,8 \\
\hline Ignorado & 2285 & 15,6 \\
\hline \multicolumn{3}{|l|}{ Tipo de parto } \\
\hline Vaginal & 8410 & 57,6 \\
\hline Cesárea & 4581 & 31,4 \\
\hline Ignorado & 1602 & 11 \\
\hline \multicolumn{3}{|l|}{ Relação com o parto } \\
\hline Antes & 16 & 0,1 \\
\hline Durante & 8 & 0,1 \\
\hline Após & 12299 & 84,3 \\
\hline Ignorado & 2270 & 15,5 \\
\hline
\end{tabular}

Fonte: DATASUS/SIM 
Tabela II - Faixa etária, escolaridade, tipo de parto e relação com o parto das genitoras das crianças que evoluíram para óbito no período neonatal. Ceará, 2005-2015.

\begin{tabular}{lcc}
\hline Variáveis maternas & $\mathbf{n = 1 4 5 9 3}$ & \% \\
\hline Faixa etária (em anos) & & 21,5 \\
$<20$ & 3135 & 51,6 \\
$20-34$ & 7533 & 10,3 \\
$>35$ & 1505 & 16,6 \\
$\quad$ Ignorado & 2420 & \\
Escolaridade & & 4,8 \\
Nenhuma & 699 & 10,9 \\
1 a 3 anos & 1592 & 20,3 \\
4 a 7 anos & 2970 & 26,8 \\
8 a 11 anos & 3900 & 6,7 \\
12 anos e mais & 983 & 30,5 \\
\hline Ignorado & 4449 & \\
\hline
\end{tabular}

Fonte: DATASUS/SIM

A TMI e seus componentes apresentaram distribuição heterogênea no estado, observando-se, em alguns municípios, valores de até 23,5 óbitos por mil NV. A análise da distribuição espacial revelou aglomerados significativos para TMI geral $(I=0,2 ; p=0,002)$ (Figura 2$)$ e o componente neonatal precoce $(I=0,14 ; p=0,006)$ (Figura 3 ).

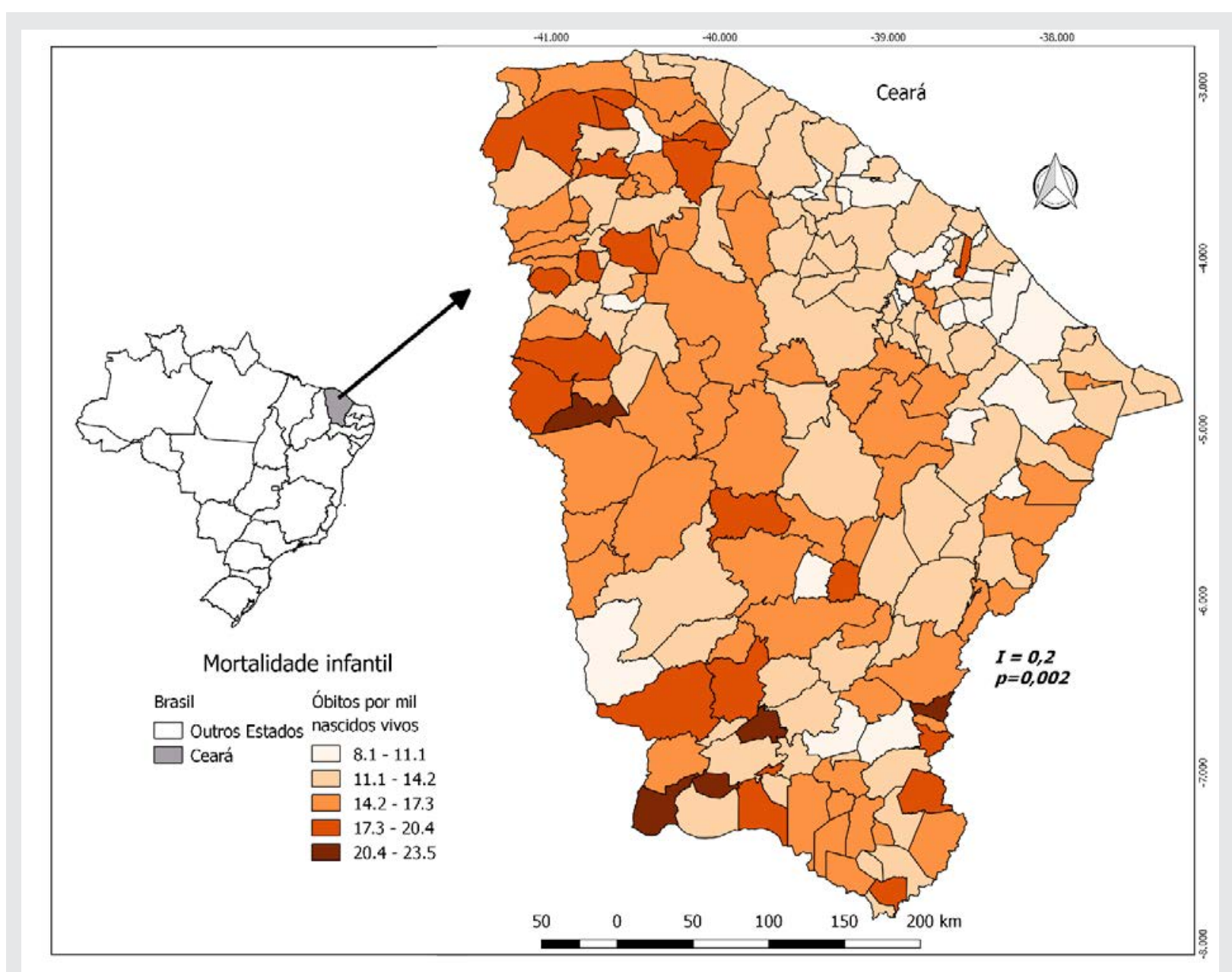

Figura 2 - Mapa da distribuição espacial da taxa de mortalidade infantil. Ceará 2005-2015. 


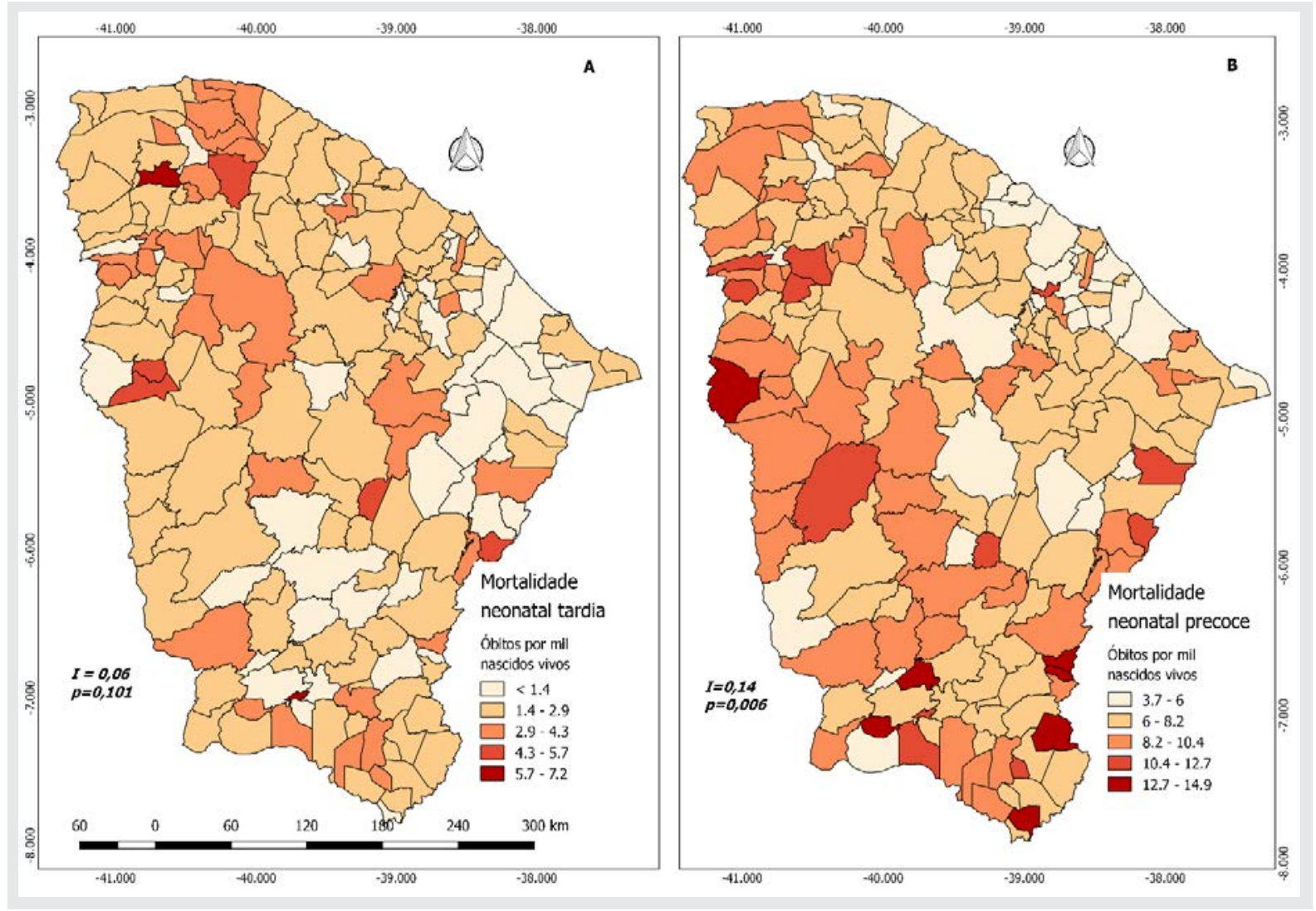

Figura 3 - Distribuição espacial da taxa de mortalidade. Ceará 2005-2015. Mapa A -Mortalidade neonatal tardia e Mapa B - Mortalidade neonatal precoce.

\section{DISCUSSÃO}

Neste estudo, encontrou-se que a grande proporção de óbitos ocorreu no período neonatal precoce, com resultados compatíveis com os de um estudo ecológico realizado em microrregiões do estado de São Paulo(14). Constata-se que muito precisa ser desenvolvido na melhoria da qualidade da atenção dispensada pela atenção primária, uma vez que a mortalidade neonatal precoce está associada à deficiência na assistência ofertada às gestantes e na atenção aos recém-nascidos ${ }^{(4)}$.

Foram observadas variações entre os municípios do estado na TMI geral e de seus componentes segundo óbito por residência no período estudado. Dados semelhantes foram encontrados em outros estudos, como um estudo realizado em Aracaju - Sergipe que observou declínio com assimetria entre os bairros da cidade, dados que devem ser considerados paras as ações de política de saúde infantil para a correta distribuição dos recursos disponíveis ${ }^{(9)}$.

Observa-se que a maioria das crianças era do sexo masculino, prematuros (menores de 37 semanas) e apresentaram peso inferior a 2500 gramas. Todos esses dados corroboram com um estudo realizado no município de Ubá, Minas Gerais, que apresentou o perfil epidemiológico da mortalidade neonatal entre os anos de 2008 a $2010^{(4)}$

Uma das explicações para a maior TMI no sexo masculino é que esses recém-nascidos apresentam menor velocidade no processo de maturação dos pulmões durante o desenvolvimento fetal, o que os levaria a um maior risco de óbito quando comparados aos recém-nascidos do sexo feminino( ${ }^{(3)}$. A prematuridade e o crescimento intrauterino restrito, que favorecem o baixo peso ao nascer, são condições evitáveis que podem ser identificadas e prevenidas durante o pré-natal. Além disso, sua associação com óbitos de menores de um ano pode indicar a existência de problemas na qualidade dessa assistência( ${ }^{(3,6)}$.

Em relação ao tipo de parto e ao momento da ocorrência do óbito, observou-se a predominância do parto normal e de óbito após o parto. Situação identificada também no Rio de Janeiro, em 2005, onde a probabilidade de morte de bebês que nasceram de parto vaginal foi 50,0\% maior quando comparada aos que nasceram de parto cirúrgico. Esses dados que devem ser interpretados com cautela, uma vez que as complicações do parto normal são mais brandas em comparação com às da cesárea, sendo recomendado pela OMS um percentual inferior a $15 \%$ de cesárias $^{(3)}$ 
Quanto à causa do óbito, segundo capítulos do CID-10, a maioria foi devido a algumas afecções originadas no período neonatal e a malformações congênitas. Segundo estudo de coorte sobre a mortalidade neonatal na pesquisa Nascer no Brasil, a causa de morte neonatal mais comum foi a prematuridade e suas complicações $(1 / 3$ dos casos), seguida pela malformação congênita $(22,8 \%)$, as infecções $(18,5 \%)$, os fatores maternos $(10,4 \%)$ e asfixia/hipóxia (7\%). Existe um maior obstáculo na redução de mortes por malformação; lembrando que algumas podem ser prevenidas, como as do sistema nervoso central, com o uso do ácido fólico, desde que seu uso seja iniciado antes da concepção(15,16).

Em relação às genitoras, observa-se que pouco mais da metade se encontrava na faixa etária entre 20 a 34 anos. Dados semelhantes foram encontrados em outros estudos, não sendo verificada associação entre idade materna e óbito neonatal ${ }^{(3,17)}$.

A escolaridade da mãe é considerada por diversos autores como um marcador da condição socioeconômica da família e reflete a desigualdade social relacionada às mortes. Neste estudo, a maioria das genitoras apresentou menos de 12 anos de estudo. A escolaridade tem influência sobre o comportamento da mãe, desde a gravidez até a criação dos filhos, estando ligada a uma adequada proteção das crianças e a melhores práticas de higiene e outros cuidados, com redução da mortalidade ${ }^{(5,18)}$.

\section{CONCLUSÃO}

O presente estudo identificou aglomerados de autocorrelação espacial para a TMI geral e o componente neonatal precoce. Apesar da redução significativa da mortalidade no período, alguns municípios apresentaram taxas acima da média do estado.

\section{CONFLITOS DE INTERESSE}

Autores declaram que não há conflitos de interesse.

\section{REFERÊNCIAS}

1. Gava C, Cardoso AM, Basta PC. Infant mortality by color or race from Rondônia, Brazilian Amazon. Rev Saúde Pública. 2017;51:1-9.

2. Caldas ADR, Santos RV, Borges GM, Valente JG, Portela MC, Marinho GL. Mortalidade infantil segundo cor ou raça com base no Censo Demográfico de 2010 e nos sistemas nacionais de informação em saúde no Brasil. Cad Saúde Pública. 2017;33(7):1-13.

3. Noronha GA, Torres TG, Kale PL. Análise da sobrevida infantil segundo características maternas, da gestação, do parto e do recém nascido na coorte de nascimento de 2005 no Município do Rio de Janeiro-RJ, Brasil. Epidemiol Serv Saúde. 2012;21(3):419-30.

4. Paula JD Jr, Lucas ES, Cunha LMC, Machado MGM, Pedrosa RL. Profile of neonatal mortality in the municipality of Ubá/ MG, Brazil (2008-2010). Rev Bras Pesqui Saúde. 2016;18(3):24-31.

5. Simões CCS. Perfis de saúde e de mortalidade no Brasil: uma análise de seus condicionantes em grupos populacionais específicos. Brasília: Organização Pan-Americana da Saúde - OPAS/OMS; 2002. 126 p.

6. Silveira MF, Santos IS, Barros AJD, Matijasevich A, Barros FC, Victora CG. Aumento da prematuridade no Brasil: revisão de estudos de base populacional. Rev Saúde Pública. 2008;42(5):957-64.

7. Brasil. Portaria n 1.459, de 24 de Junho de 2011. Institui, no âmbito do Sistema Único de Saúde - SUS - a Rede Cegonha. Diário Oficial da União; Brasília, 27 Jun. 2011; Seção 1.

8. Batista M Filho, Cruz RSBLC. A saúde das crianças no mundo e no Brasil. Rev Bras Saúde Mater Infant. 2015;15(4):451-4.

9. Carvalho RAS, Santos VS, Melo CM, Gurgel RQ, Oliveira CCC. Inequalities in health: living conditions and infant mortality in Northeastern Brazil. Rev Saúde Pública. 2015;49:1-9.

10. Governo do Brasil. Mortalidade infantil continua em queda no Brasil. [Internet]. 2016. [acesso em 2017 Ago 26]. Disponível em: http://www.brasil.gov.br/editoria/saude/2016/11/mortalidade-infantil-continua-em-queda-no-brasil. 
11. Secretaria de Saúde (CE). Informe Epidemiológico Mortalidade Infantil [Internet]. 2014. [acesso em 2017 Ago 26]. Disponível em: http://ww9.saude.ce.gov.br/wp-content/uploads/sites/9/2018/06/informe_mortalidade_infantil_ set_2014.pdf.

12. Secretaria de Saúde (CE), Coordenadoria de Promoção e Proteção à Saúde, Núcleo de Vigilância Epidemiológica. Boletim Epidemiológico de Mortalidade Materna, Infantil e Fetal [Internet]. 2017. [acesso em 2018 Jun 20]. Disponível em: http://www.saude.ce.gov.br/index.php/boletins?download...mortalidade-materna-infantil.

13. Pedrosa NL, Santos VF, Paiva SS, Galvão MTG, Almeida RLF, Kerr LRFS. Specialized care for people with AIDS in the state of Ceara, Brazil. Rev Saúde Pública. 2015;49:1-7.

14. Almeida MCS, Gomes CMS, Nascimento LFC. Análise espacial da mortalidade neonatal no estado de São Paulo, 2006-2010. Rev Paul Pediatr. 2014;32(4):374-80.

15. Arruda TAM, Amorim MMR, Souza ASR. Mortalidade determinada por anomalias congênitas em Pernambuco, Brasil, de 1993 a 2003. Rev Assoc Med Bras. 2008;54(2):122-6.

16. Lansky S, Friche AAL, Silva AAM, Campos D, Bittencourt SDA, Carvalho ML, et al. Pesquisa Nascer no Brasil: perfil da mortalidade neonatal e avaliação da assistência à gestante e ao recém-nascido. Cad Saúde Pública. 2014;30(Supl 1):S192-207.

17. Lima JC, Mingarelli AM, Segri NJ, Zavala AAZ, Takano OA. Population-based study on infant mortality. Ciênc Saúde Colet. 2017;22(3):931-9.

18. Morais OL Neto, Barros MBA. Fatores de risco para mortalidade neonatal e pós-neonatal na Região CentroOeste do Brasil: linkage entre bancos de dados de nascidos vivos e óbitos infantis. Cad Saúde Pública. 2000;16(2):477-85.

\section{Endereço para correspondência:}

Ana Nery Melo Cavalcante

Universidade de Fortaleza - UNIFOR

Programa de Pós-Graduação em Saúde Coletiva

Av. Washington Soares, 1321/ Bloco S/ Sala 1

Bairro: Edson Queiroz

CEP: 60811-905 - Fortaleza - CE - Brasil

E-mail: ananery.melo@unifor.br 